\title{
Tableau Recycling
}

\author{
Angelika Mader * \\ Technische Universität München \\ Arcisstr. 21 \\ W - 8000 München 2 \\ Germany \\ e-mail: mader@informatik.tu-muenchen.de
}

\begin{abstract}
In this paper we improve a model checking algorithm based on the tableau method of Stirling and Walker. The algorithm proves whether a property expressed in the modal mu-calculus holds for a state in a finite transition system. It makes subsequent use of subtableaux which were calculated earlier in the proof run. These subtableaux are reduced to expressions. Examples show that both size of tableaux and execution time of the algorithm are reduced.
\end{abstract}

\section{Introduction}

The modal mu-calculus is an active area of research. It stands in the tradition of Hoare logic, Dynamic logic, Process logic, and linear and branching time logics [S]. Model checking in the modal mu-calculus plays a part in verification of parallel processes with both finite [CS1] and infinite state spaces [BS], and finds application in preorder models [CS2] and in Petri nets [B].

The main approaches are symbolic model checking [BC] [EFT], model checkers based on the fixpoint induction principle [EL], and tableau based model checkers as in [SW] [C]. An advantage of the latter is its ability to deal also with infinite state spaces. In comparison to the approximation techniques they work locally, i.e. they do not determine the set of all states satisfying a property, but prove a modal formula only for one state. Unfortunately the attractiveness of tableau methods suffers by their complexity. A main reason for this is that these model checkers do not make subsequent use of subresults.

This paper presents a method whereby a tableau based model checker for the full rnodal mu-calculus can recycle subtableaux, which have been calculated earlier in the model checking algorithm. An implementation of these ideas has confirmed an impressive improvement in execution speed in a variety of examples.

The following section introduces briefly the modal mu-calculus and its semantics. In section 3 the underlying standard tableau model checker is described. The motivating

supported by Siemens AG, Corporate Research and Development 
ideas of tableau recycling and the necessary notions are contained in section 4 . Sections 5 and 6 present the algorithm and the proofs of its correctness and completeness. Some surprising examples can be found in section 7 . Section 8 concludes this paper.

\section{The Modal Mu-Calculus}

This section gives a brief introduction to the modal mu-calculus. For more details see [S].

The syntax of the modal mu-calculus is defined with respect to a set $\mathcal{Q}$ of atomic propositions including true and false, a finite set $\mathcal{L}$ of action labels and a denumerable set $\mathcal{Z}$ of propositional variables. A formula of the modal mu-calculus is an expression of the form:

$$
A::=Z|Q| \neg A|A \wedge A|[a] A \mid \nu Z . A
$$

where $Z \in \mathcal{Z}, Q \in \mathcal{Q}$ and $a \in \mathcal{L}$, and where in $\nu Z$.A every free occurence of $Z$ in $A$ falls under an even number of negations. The standard conventions for the derived operators are:

$A_{1} \vee A_{2}:=\neg\left(\neg A_{1} \wedge \neg A_{2}\right)$

$\langle a\rangle A \quad:=\neg[a] \neg A$

$\mu Z . A \quad:=\neg \nu Z . \neg A[\neg Z / Z]$.

Formulae of the modal mu-calculus with the set $\mathcal{L}$ of action labels are interpreted relative to a labelled transition system $\mathcal{T}=(\mathcal{S},\{\stackrel{a}{\rightarrow} \mid a \in \mathcal{L}\})$, where $\mathcal{S}$ is a finite set of states and $\stackrel{a}{\rightarrow} \subseteq \mathcal{S} \times \mathcal{L} \times \mathcal{S}$ for every $a \in \mathcal{L}$ a binary relation on states. A valuation function $\mathcal{V}$ assigns to every atomic proposition $Q$ in $\mathcal{Q}$ (and propositional variable $Z$ in $\mathcal{Z}$ ) a set of states $\mathcal{V}(Q) \subseteq \mathcal{S}(\mathcal{V}(Z) \subseteq \mathcal{S}$ ) meaning that the proposition $Q$ (variable $Z$ ) holds for every state in $\mathcal{V}(Q)(\mathcal{V}(Z))$. The pair $\mathcal{T}$ and $\mathcal{V}$ is called a model of the mu-calculus. The semantics of each mu-calculus formula $A$ is the set of states $\|A\|_{\mathcal{V}}^{T}$ defined inductively as follows:

$$
\begin{aligned}
& \|Z\|_{\mathcal{V}}^{\mathcal{T}} \quad=\mathcal{V}(Z) \\
& \|Q\|_{\mathcal{V}}^{\tau} \quad=\mathcal{V}(Q) \\
& \|\neg A\|_{\nu}^{\tau} \quad=\mathrm{S}-\|A\|_{\nu}^{\tau} \\
& \left\|A_{1} \wedge A_{2}\right\|_{\nu}^{T}=\left\|A_{1}\right\|_{\nu}^{\mathcal{T}} \cap\left\|A_{2}\right\|_{\nu}^{T} \\
& \|[a] A\|_{v}^{T}=\left\{s \in \mathcal{S} \mid \forall s^{\prime} . \text { if } s \stackrel{a}{\rightarrow} s^{\prime} \text { then } s^{\prime} \in\|A\|_{V}^{\mathcal{T}}\right\} \\
& \|\nu Z . A\|_{\mathcal{V}}^{\mathcal{T}}=\bigcup\left\{\mathcal{S}^{\prime} \subseteq \mathcal{S} \mid \mathcal{S}^{\prime} \subseteq\|A\|_{\mathcal{V}\left[\mathcal{S}^{\prime} / z\right]}^{\tau}\right\}
\end{aligned}
$$

\section{A Standard Tableau Model Checker}

This section sketches a standard tableau method based on the model checker of Stirling $\&$ Walker [SW]. The notation used here is a mixture of the notations of Cleaveland [C] and Stirling \& Walker [SW]. Some additional notions are necessary.

The modal mu-calculus is extended by a set of propositional constant symbols. Let 
$U, U_{1} \ldots$ range over these symbols. A definition is a declaration $U=A$, where $U$ is a constant symbol and $A$ a formula of the modal mu-calculus which may contain constant symbols. A definition list $\Delta$ consists of a sequence of definitions $\left(U_{1}=\right.$ $\left.A_{1}\right) \ldots\left(U_{n}=A_{n}\right)$. It fulfills the requirement that every $U_{j}$ appearing in $A_{i}$ is defined before $U_{i}$, i.e. $j \leq i$, and that $U_{i} \neq U_{j}$ for $i \neq j$. For $\Delta$ being such a definition list the function $\Delta\left(U_{i}\right)=A_{i}$ is declared. A hypothesis is an expression of the form $s \in U$, where $s$ is a state of a transition system and $U$ a constant symbol. Hypotheses are collected in a hypothesis set $H$. A sequent $H \vdash_{\Delta} s \in A$ expresses that the formula $A$ is valid at the state $s$ with respect to the hypotheses of $H$. We drop empty $\Delta s$ and $H$ s.

The model checker here is tableau based. This corresponds to a top-down proof method, starting with the intended conclusion and reducing it stepwise to (atomic) premisses. The rules for a tableau method are inverse to the usual rules of natural deduction. Here we take the conclusions and premisses to be sequents. The root sequent $\vdash s \in A$ contains the state $s$ and the modal property $A$, which we want to prove for this state. The root sequent has an empty hypothesis set and an empty definition list. The rules of the tableau system are:

$$
\begin{aligned}
& \text { 1) } \frac{H \vdash_{\Delta} s \in \neg \neg A}{H \vdash_{\Delta} s \in A} \\
& \text { 2) } \frac{H \vdash_{\Delta} s \in A \wedge B}{H \vdash_{\Delta} s \in A \quad H \vdash_{\Delta} s \in B} \\
& \text { 3) } \frac{H \vdash_{\Delta} s \in \neg(A \wedge B)}{H \vdash_{\Delta} s \in \neg A} \\
& \text { 4) } \frac{H \vdash_{\Delta} s \in \neg(A \wedge B)}{H \vdash_{\Delta} s \in \neg B} \\
& \text { 5) } \frac{H \vdash_{\Delta} s \in[a] A}{H \vdash_{\Delta} s_{1} \in A \ldots H \vdash_{\Delta} s_{n} \in A} \quad\left\{s_{1}, \ldots, s_{n}\right\}=\left\{s^{\prime} \mid s \stackrel{a}{\rightarrow} s^{\prime}\right\} \\
& \text { 6) } \frac{H \vdash_{\Delta} s \in \neg[a] A}{H \vdash_{\Delta} s^{\prime} \in \neg A} \quad s \stackrel{a}{\rightarrow} s^{\prime} \\
& \text { 7) } \frac{H r_{\Delta} s \in \nu Z . A}{H r_{\Delta^{\prime}} s \in U} \quad \Delta^{\prime}=\Delta \cdot(U=\nu Z . A) \\
& \text { 8) } \frac{H \vdash_{\Delta} s \in \neg \nu Z . A}{H \vdash_{\Delta^{\prime}} s \in U} \quad \Delta^{\prime}=\Delta \cdot(U=\neg \nu Z . A) \\
& \text { 9) } \frac{H \vdash_{\Delta} s \in U}{H^{\prime} \vdash_{\Delta} s \in A[Z:=U]}(s \in U) \notin H, \Delta(U)=\nu Z . A, H^{\prime}=H \cup\{s \in U\} \\
& \text { 10) } \frac{H \vdash_{\Delta} s \in U}{H^{\prime} \vdash_{\Delta} s \in \neg A[Z:=\neg U]}(s \in U) \notin H, \Delta(U)=\neg \nu Z . A, H^{\prime}=H \cup\{s \in U\}
\end{aligned}
$$

A proof tree is constructed by applying the rules to the root sequent, and then to its successors etc. The proof tree is maximal if no rule is applicable to a leaf sequent. Such a maximal proof tree is called a tableau. A tableau is successful, if all its leaves are successful. A leaf sequent $H \vdash_{\Delta} s \in B$ is successful, if it satisfies one of the properties (i)-(iv): 
(i) $B=Q$ and $s \in \mathcal{V}(Q)$

(ii) $B=\neg Q$ and $s \notin \mathcal{V}(Q)$

(iii) $B=[a] C$

(iv) $B=U$ and $\Delta(U)=\nu Z$.C and $(s \in U) \in H$ (i') $B=Q$ and $s \notin \mathcal{V}(Q)$

(ii') $B=\neg Q$ and $s \in \mathcal{V}(Q)$

(iii') $B=\langle a\rangle C$

(iv') $B=U$ and $\Delta(U)=\neg \nu Z . C$ and $(s \in U) \in H$

If one of the dual forms (i')-(iv') of these requirements holds for a leaf sequent, then it is not successful. In an unsuccessful tableau there is at least one leaf, which is not successful.

For later considerations the definition of a computation tree is also neccessary. A model checker algorithm based on the tableau rules builds a tree starting with $\vdash s \in A$ as root sequent and applying nondeterministically the rules. When a leaf fails the algorithm has to use backtracking techniques to try other paths. It will build up this tree until it is sure that a sequent has a successful subtableau or not. In the first case the tree includes a successful tableau, in the second case it is not necessary that the tree contains any maximal proof tree. We call a tree created by such a model checker algorithm a computation tree, if it sufficient to decide, whether there exists a successful tableau or not.

Note that such a computation tree determines an "and-or-tree", if the successful leaves are identified with true, the unsuccessful ones with false, the nondeterministic branching as disjunction and the deterministic branching as conjunction. It can be evaluated to true, iff the computation tree contains a successful tableau.

For simplicity of notation in this paper the definition lists $\Delta, \Delta^{\prime}$ are considered to be global for all different subtrees of one computation tree, i.e. there are no two different definition lists (in two different subtrees) $\Delta_{1}, \Delta_{2}$ and for any $U, \Delta_{1}(U) \neq \Delta_{2}(U)$.

\section{$4 \quad$ Tableau Recycling}

This section starts by describing a basic source of inefficiency in the standard model checker. Then it considers how the efficiency might be improved, and finally means for an algorithm are presented that gives a significant gain in efficiency.

The basic problem with the standard model checker is that it does not store any intermediate results (subresults). It is possible that it proves the same formula for the same state arbitrarily often, as the following example will show:

$\vdash s_{1} \in \Phi$

$\Phi=\nu X \cdot[a]\langle b\rangle X$

informal meaning: "every a-successor

has a b-successor for which

this property holds, recursively."

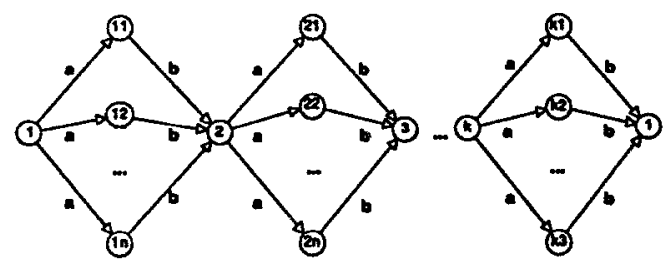

Fig. 1. model with tableau of exponentional size 
Here the formula $\Phi$ is proved $n$ times for the state $s_{2}, n^{2}$ times for the state $s_{3}, \ldots, n^{k-1}$ times for the state $s_{k}$. The number of nodes in the computation tree is exponential with respect to the length $k$ of this transition system.

Moreover, a look at computation trees shows that in many cases, different subtableaux of a state and a formula are very similar.

The most obvious way to improve efficiency would be just to store the information "the sequent $H \vdash s \in \Phi$ has a successful (or no successful) subtableau", and use it whenever the same state and formula appear again in the computation tree.

Unfortunately this idea is too simple:

- The first obstacle is that the constants used in different subtableaus have different names. It therefore does not happen that exactly the same sequent appears twice. A notion of equivalence will help to solve this problem.

- Secondly the hypothesis sets can differ, even when formulae and states are identical. It is obvious that the shapes of the computation subtrees differ accordingly, since they depend on the hypothesis sets. Therefore in order to recycle a computation subtree of a similar sequent with even a slightly different hypothesis set, one has to store also the shape of the computation subtree. It turns out that the shape of such a subtree can be reduced to an expression which is sufficient for the derivation of all useful information.

A cornerstone of the tableau recycling algorithm is a notion of equivalence, which allows different sequents in a computation tree to be compared. The basis for this is the definition of equivalent constants, which have different names but identify syntactically the same formula. In the following let for $Z_{1}, \ldots, Z_{n}$ being the free variables in $\Phi$, denote $\Phi\left(U_{j_{1}}, \ldots U_{j_{n}}\right)=\Phi\left[U_{j_{1}} / Z_{1}, \ldots U_{j_{n}} / Z_{n}\right]$, meaning that every occurence of $Z_{i}$ in $\Phi$ is substituted by $U_{j i}$.

\section{DEFINITION 1 (Equivalence)}

(1) A formula $\Phi\left(U_{j_{1}}, \ldots U_{j_{n}}\right)$ is equivalent to the formula $\Phi\left(U_{k_{1}}, \ldots U_{k_{n}}\right)$, denoted $\Phi\left(U_{j_{1}}, \ldots U_{j_{n}}\right) \sim \Phi\left(U_{k_{1}}, \ldots U_{k_{n}}\right)$, iff for $1 \leq i \leq n$ all constants $U_{j i} \sim U_{k_{i}}$.

(2) A constant $U_{1}$ is equivalent to the constant $U_{2}$, denoted $U_{1} \sim U_{2}$, iff $U_{1}=U_{2}$ or $\Delta\left(U_{1}\right) \sim \Delta\left(U_{2}\right)$.

Note that this equivalence is essentially alpha-conversion with respect to constants. The main insight now is that equivalent sequents can have identical computation subtrees.

We will now show how the shape of a computation tree can be reduced to an expression. 
DEFINITION 2 ( Hypothesis Tree)

A hypothesis tree is an expression of the form:

$$
H T::=\text { true } \mid \text { false } \mid \text { unknown }|Y| Y_{;} H T\left|\bigvee_{i \in I} H T_{i}\right| \bigwedge_{i \in I} H T_{i}
$$

where $I$ is a finite index set and $Y$ is a hypothesis, e.g. $s \in U$.

If the termination behavior of all paths of the computation tree is known, it is determined whether there is a successful subtableau or not. Some of the paths terminate with the rules (i)-(iii) and their dual forms. This kind of termination is independent of the hypothesis sets. The other paths terminate with rule (iv) and its dual form. In this case the kind of termination depends on the hypothesis set and whether for the terminal sequent $H^{\prime} \vdash_{\Delta} s \in U$ the constant $U$ stands for a maximal or negated maximal (minimal) fixpoint formula.

The reduction of a computation tree to a hypothesis tree reflects this idea: transform the computation tree to an "and-or tree", but leave all hypotheses in it.

DEFINITION 3 ( Reduction to a Hypothesis Tree)

Reduce a computation tree to a hypothesis tree in the following way:

- Replace every leaf terminating with rules (i)-(iii) or their dual forms by true or false respectively.

- Replace every leaf terminating with a hypothesis which is not contained in the root hypothesis set by true if the leaf is successful or false otherwise.

- Replace every nondeterministic branching by a disjunction. If not all of the nondeterministic rules were applied, extend this disjunction by a leaf unknown. The number of all possible nondeterministic rules is called the arity of the disjunction.

- Replace every deterministic branching by a conjunction. If not all of the deterministic rules were applied, extend this conjunction by a leaf unknown. The number of all possible deterministic rules is called the arity of the conjunction.

- Drop all sequents which are not of the form $H \vdash_{\Delta} s \in U$, and drop all hypothesis sets.

\section{DEFINITION 4 (Evaluation of a Hypothesis Tree)}

The function eval evaluates a hypothesis tree HT together with a hypothesis set $H$ to eval $(H T, H) \in\{$ true, false, unknown $\}$ in the following way:

- substitute every hypothesis $s \in U$ in $H T$ which is contained in $H$ by true if $\Delta(U)=\nu X . A$, else by false .

- substitute every hypothesis which is not contained in $H$ by unknown.

- Evaluate this tree with the following rules which are extended in the obvious way for indexed conjunction and disjunction: 


$\left.\begin{array}{|l|lll|}\hline \vee & \text { true } & \text { false } & \text { unknown } \\ \hline \text { true } & \text { true } & \text { true } & \text { true } \\ \text { false } & \text { true } & \text { false } & \text { unknown } \\ \text { unknown } & \text { true } & \text { unknown unknown }\end{array}\right]\left[\begin{array}{llll}\wedge & \text { true } & \text { false } & \text { unknown } \\ \hline \text { true } & \text { true } & \text { false } & \text { unknown } \\ \text { false } & \text { false } & \text { false } & \text { false } \\ \text { unknown } & \text { unknownfalse } & \text { unknown } \\ \hline\end{array}\right.$

The operator ";" is treated as follows:

$$
\begin{array}{ll}
\text { eval ( unknown } & ; H T, H)=\operatorname{eval}(H T, H) \\
\text { eval }(\text { true } & ; H T, H)=\text { true } \\
\text { eval }(\text { false } & ; H T, H)=\text { false }
\end{array}
$$

The following definition reflects the idea that hypothesis trees with equivalent roots particulary have a common structure. Combining two hypothesis trees then means that the common structure is identified and extended by both non common structures parts.

\section{DEFINITION 5 (Combination of Hypothesis Trees)}

Consider two sequents $H_{1} \downarrow_{\Delta} s \in U_{1}$ and $H_{2} \downarrow_{\Delta} s \in U_{2}, U_{1} \sim U_{2} . \tau_{1}$ is the hypothesis tree constructed from a computation tree of the first sequent, $\tau_{2}$ from the second one. Let $\tau_{3}=\tau_{1}$ o $\tau_{2}$ be the combination of $\tau_{1}$ and $\tau_{2}$ such that

- The root of $\tau_{3}$ is the root of $\tau_{1}$.

- If $t \in U_{1}^{\prime}$ is a successor of $s \in U_{1}$ with the subtree $\tau_{1}^{\prime}$ and $t \in U_{2}^{\prime}$ is a successor of $s \in U_{2}$ with the subtree $\tau_{2}^{\prime}$ such that $U_{1}^{\prime} \sim U_{2}^{\prime}$,

then $\tau_{1}^{\prime} \circ \tau_{2}^{\prime}$ is a direct subtree of the root of $\tau_{3}$.

- If $t \in U_{1}^{\prime}$ is a successor of $s \in U_{1}$ with the subtree $\tau_{1}^{\prime}$ and there is no successor $t \in U_{2}^{\prime}$ of $s \in U_{2}$ such that $U_{1}^{\prime} \sim U_{2}^{\prime}$,

then $\tau_{1}^{\prime}$ is a direct subtree of the root of $\tau_{3}$. (symmetrically for $t \in U_{2}^{\prime}$ )

- If the successors of $s \in U_{1}$ and $s \in U_{2}$ are disjunctions (neccessarily with the same arity) then the successor of the root of $\tau_{3}$ is also a disjunction with the same arity and those branches are combined pairwise which correspond to the same rule applied at this place in the computation tree. Branches of one disjunction which have no corresponding branch of the other disjunction appear directly in the combined disjunction. If the arity of the disjunction and the number of branches are equal all leaves unknown in this disjunction are dropped. (analogously for conjunction)

\section{The Tableau Recycling Model Checker}

The standard tableau model checker is extended by a set of hypothesis trees $\mathcal{H} T$. In the beginning $\mathcal{H T}$ is initialized with the empty set. 
Every time, when a computation tree is built up for a sequent $H \vdash_{\Delta} s \in U$, the hypothesis tree $\tau$ is derived and inserted in $\mathcal{H} T$. If there already exists a hypothesis tree $\tau_{1}$ in $\mathcal{H} \mathcal{T}$ with a equivalent root $s \in U^{\prime}, U \sim U^{\prime}$, the combined hypothesis tree $\tau \circ \tau_{1}$ is added to $\mathcal{H T}$ replacing $\tau_{1}$.

The tableau rules 9 ) and 10) are extended by a further requirement:

9')

$$
\frac{H \vdash_{\Delta} s \in U}{H^{\prime} \vdash_{\Delta} s \in A[Z:=U]}(s \in U) \notin H, \Delta(U)=\nu Z . A, H^{\prime}=H \cup\{s \in U\}
$$

and there is no $\tau \in \mathcal{H T}$ such that the root $s \in U^{\prime}, U \sim U^{\prime}$ and eval $(\tau, H) \in\{$ true, false $\}$;

10')

$$
\frac{H \vdash_{\Delta} s \in U}{H^{\prime} \vdash_{\Delta} s \in \neg A[Z:=\neg U]}(s \in U) \notin H, \Delta(U)=\neg \nu Z . A, H^{\prime}=H \cup\{s \in U\}
$$

and there is no $\tau \in \mathcal{H T}$ such that the root $s \in U^{\prime}, U \sim U^{\prime}$ and eval $(\tau, H) \in\{$ true, false $\}$;

In addition to the conditions (i)-(iv) stated in section 3 a leaf sequent $H r_{\Delta} s \in U$ of a tableau constructed by by rules 1)-8), $9^{\prime}$ ) and $10^{\prime}$ ) is also successful if it fulfills the following property:

(v) There exists a $\tau \in \mathcal{H} T$ such that the root $s \in U^{\prime}, U \sim U^{\prime}$ and eval $(\tau, H)=$ true.

The number of elements in the set $\mathcal{H} \mathcal{T}$ is bounded: for every state in the transition system $\mathcal{T}$ and every fixpoint operator in the root formula there is at most one hypothesis tree contained in $\mathcal{H T}$.

The example on the next page should clarify the algorithm.

\section{Correctness and Completeness}

In this section it will be shown that the recycling model checker produces the same results as the model checker from Stirling \& Walker [SW]. Then correctness and completeness of the model checker presented here follow from the correctness and completeness proved in [SW].

The way of argumentation is as follows: the propositions here are valid for both versions, the standard tableau model checker and the recycling one. First some properties of tableaux and computation trees with similar root sequents are stated. In the remainder it is shown that the evaluation of a hypothesis tree corresponds to the result derived from a computation tree.

As a first step the notion of equivalence given in definition 1 must be extended to hypothesis sets and sequents. 

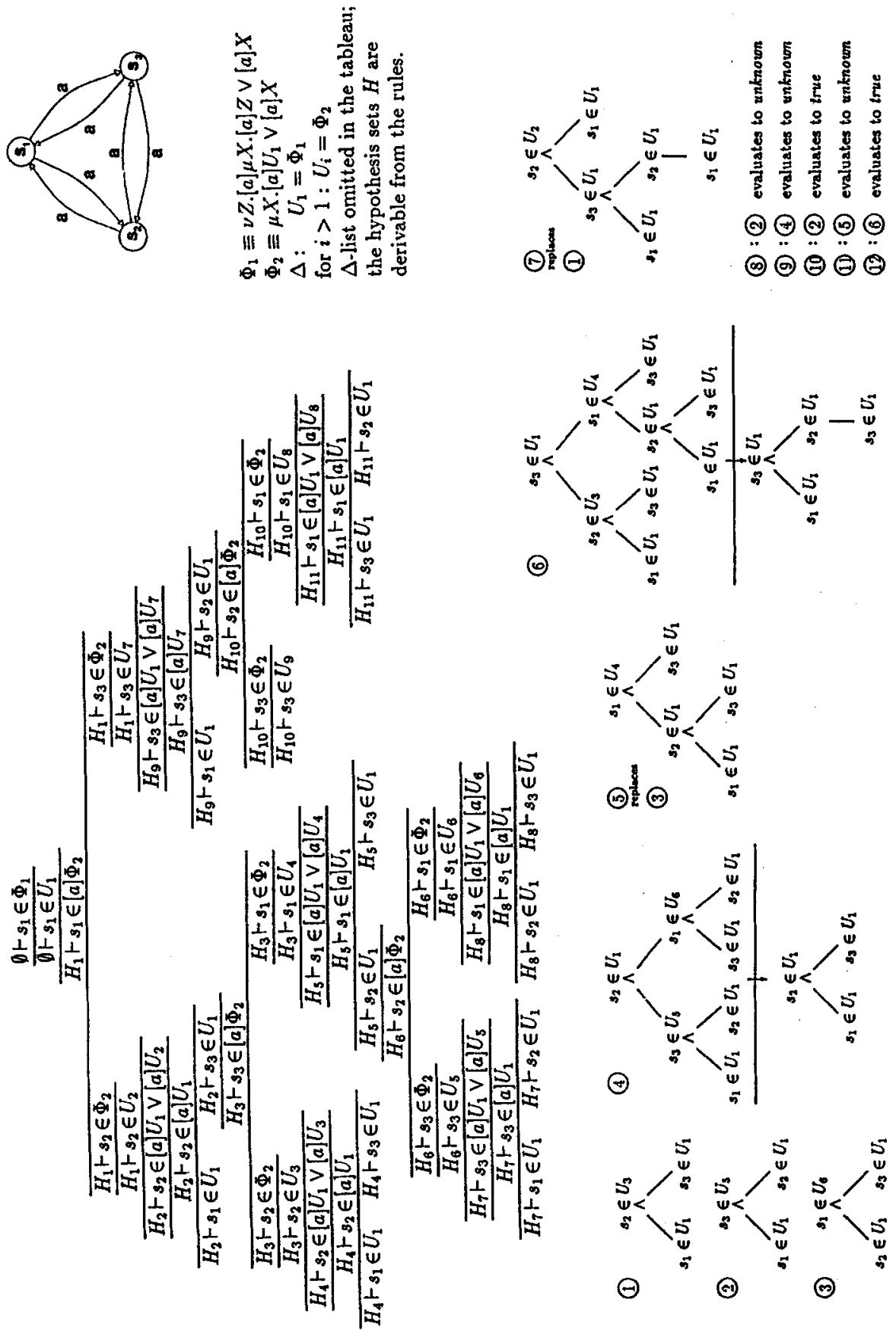

蛋

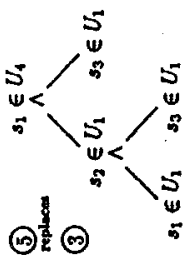

g

灵
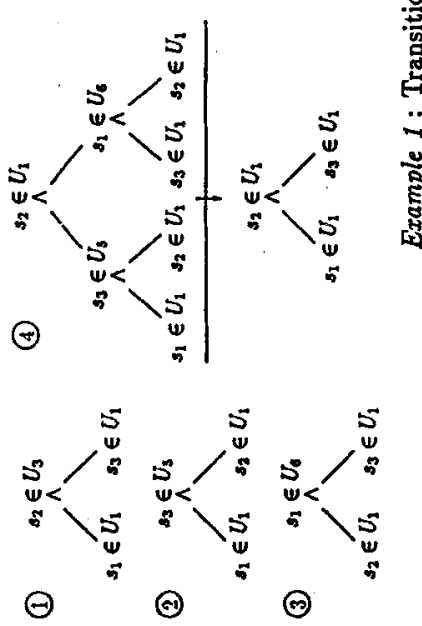


\section{DEFINITION 6 ( Equivalence)}

(3) A hypothesis set $H_{1}$ is smaller than a hypotheses set $H_{2}$ with respect to the equivalent constants $U_{1}, U_{2}$, denoted $H_{1} \leq_{U_{1}, U_{2}} H_{2}$, iff

- for every hypothesis $\left(s \in U_{1}\right) \in H_{1}$ the hypothesis $\left(s \in U_{2}\right)$ is contained in $H_{2}$, and

- with $\Delta\left(U_{1}\right)=\Phi\left(U_{j_{1}}, \ldots U_{j_{n}}\right)$ and $\Delta\left(U_{2}\right)=\Phi\left(U_{k_{1}}, \ldots U_{k_{n}}\right)$ for every $1 \leq i \leq n$ holds $H_{1} \leq_{U_{j_{i}}, U_{k_{i}}} H_{2}$.

(4) Two hypothesis sets $H_{1}$ and $H_{2}$ are equivalent with respect to the equivalent constants $U_{1}, U_{2}$, denoted $H_{1} \sim U_{1}, U_{2} H_{2}$, iff $H_{1} \leq_{U_{1}, U_{2}} H_{2}$ and $H_{2} \leq_{U_{1}, U_{2}} H_{1}$.

(5) Two sequents $H_{1} \vdash_{\Delta} s \in \Phi\left(U_{j_{1}}, \ldots U_{j_{n}}\right)$ and $H_{2} \vdash_{\Delta} s \in \Phi\left(U_{k_{1}}, \ldots U_{k_{n}}\right)$ are equivalent, iff for $1 \leq i \leq n U_{j_{i}} \sim U_{k_{i}}$, and $H_{1} \sim U_{j_{i}}, V_{k_{i}} H_{2}$.

PROPOSITION 1 (Equivalent Sequents have the same Subtableaux)

Suppose $\tau_{1}$ is a computation tree with the root sequent $H_{1} \vdash_{\Delta_{1}} s \in \Phi_{1}$, and $H_{2} \vdash_{\Delta_{2}} s \in \Phi_{2}$ is an equivalent sequent. Then there exists a computation tree $\tau_{2}$ of $H_{2} \vdash s \in \Phi_{2}$ with the same branching structure as $\tau_{1}$ such that every node of $\tau_{2}$ is labelled by a sequent which is equivalent to the sequent of corresponding node of $\tau_{1}$.

Proof: by induction in the structure of $\tau_{1}$

Induction hypothesis: $s e q_{1} \equiv H_{i} \vdash_{\Delta_{i}} t \in \Phi_{i}$ in $\tau_{1}$ and $s e q_{2} \equiv H_{j} \vdash_{\Delta_{j}} t \in \Phi_{j}$ in $\tau_{2}$ are equivalent sequents.

Base case: the induction hypothesis is true for the root sequents of $\tau_{1}$ and $\tau_{2}$ by assumption.

Induction step: argumentation about the applicable rules

- leaf sequents

If $s e q_{1}$ is a leaf sequent and fulfills one of the requirements (i) - (iii) or their dual forms, then $\Phi_{i}=\Phi_{j}$ and there is no rule applicable to seq 2 .

The more interesting case is if the leaf sequent $s e q_{1}$ fulfills (iv) or its dual form. Here for $\Phi_{i}=U_{i}$ the hypothesis $t \in U_{i}$ is contained in $H_{i}$. Since for $\Phi_{j}=U_{j}$ $U_{i} \sim U_{j}$ and $H_{i} \sim U_{i}, U_{j} H_{j}$ the hypothesis $t \in U_{j}$ must be contained in $H_{j}$. Therefore also $s e q_{2}$ is also a leaf sequent.

- One of the rules 1) - 4) is applicable to the sequent $\operatorname{seq}_{1}$.

As equivalent formulae have equivalent structure, the same rule can be applied to $s e q_{2}$. As equivalent formulae have also equivalent subformulae the successor $s e q_{2}^{\prime}$ of $s e q_{2}$ is equivalent to the successor $s e q_{1}^{\prime}$ of $s e q_{1}$.

- Rule 5) or 6) is applicable to $s e q_{1}$.

$\Phi_{i}=[a] \Phi_{i}^{\prime}\left(\right.$ or $\left.\Phi_{i}=\neg[a] \Phi_{i}^{\prime}\right)$, hence also $\Phi_{j}=[a] \Phi_{j}^{\prime}\left(\right.$ or $\left.\Phi_{j}=\neg[a] \Phi_{j}^{\prime}\right)$ and $\Phi_{i}^{\prime} \sim \Phi_{j}^{\prime}$. All a-successors of the state $s$ depend only on the transition system. Therefore the same rule can be applied to $s e q_{2}$ and the successor sequents of $s e q_{2}$ contain the same states, equivalent hypothesis sets and equivalent formulae as the sequent successors of seq $_{1}$. 
- One of the rules 7) or 8) is applicable to $s e q_{1}$.

A new constant $U_{i}^{\prime}$ is generated in $\tau_{1}$. As the same rule must be applicable to $s e q_{2}$, also a new constant $U_{j}^{\prime}$ is generated in $\tau_{2}$ with $\Delta_{i}^{\prime}\left(U_{i}^{\prime}\right) \sim \Delta_{j}^{\prime}\left(U_{j}^{\prime}\right)$. Therefore the successor sequent $H_{i} \vdash_{\Delta_{i}^{\prime}} t \in U_{i}^{\prime}$ is equivalent to $H_{j} \vdash_{\Delta_{j}^{\prime}} t \in U_{j}^{\prime}$.

- Rule 9) or 10) is applied to $\operatorname{seq}_{1}$.

Here $H_{i} \vdash_{\Delta_{i}} t \in U_{i}$ and $\left(t \in U_{i}\right) \notin H_{i}$. For $H_{j} \vdash_{\Delta_{j}} t \in U_{j}$ holds by induction hypothesis $U_{i} \sim U_{j}$ and $H_{i} \sim H_{j}$. Therefore $\left(t \in U_{j}\right) \notin H_{j}$. For the successor sequent holds

$H_{i} \cup\left\{t \in U_{i}\right\}=H_{i}^{\prime} \sim H_{j}^{\prime}=H_{j} \cup\left\{t \in U_{j}\right\}$ and as

$\sigma X . A\left(X, U_{i_{1}}, \ldots, U_{i_{n}}\right)=\Delta_{i}\left(U_{i}\right) \sim \Delta_{j}\left(U_{j}\right)=\sigma X . A\left(X, U_{j_{1}}, \ldots, U_{j_{n}}\right)$ the successor sequents are equal.

\section{PROPOSITION 2 ( Size of the Computation Trees )}

Consider a sequent $H_{1} \vdash_{\Delta_{1}} s \in \Phi\left(U_{1}, \ldots U_{n}\right)$ having $\tau_{1}$ as computation tree, and a sequent $H_{2} \vdash_{\Delta_{1}} s \in \Phi\left(U_{1}, \ldots U_{n}\right)$ with $H_{1} \leq_{U_{i}, U_{i}} H_{2}$ for all $1 \leq i \leq n$. Then $H_{2} \vdash_{\Delta_{2}} s \in \Phi\left(U_{1}, \ldots U_{n}\right)$ has a computation tree $\tau_{2}$ which $s$ contained in $\tau_{1}$ as subtree, such that (up to the hypothesis sets).

Proof: by induction in the structure of $\tau_{1}$ omitted in this version

\section{PROPOSITION 3 (Combination of Hypothesis Trees)}

Let $\tau_{1}$ be the hypothesis tree derived from a computation tree of $H_{1} \vdash_{\Delta} s \in U$ and $\tau_{2}$ be the hypothesis tree derived from a computation tree of $\mathrm{H}_{2} \mathrm{r}_{\Delta} s \in U$.

Then there exists a hypothesis set $H_{3}$ with $H_{1} \cap H_{2} \subseteq H_{3} \subset H_{1} \cup H_{2}$, and $\tau_{1} \circ \tau_{2}$ as in definition 5 is the hypothesis tree derived from a computation tree of $H_{3} \vdash_{\Delta} s \in U$.

Proof: by induction in the structure of $\tau_{1}$ and $\tau_{2}$

omitted in this version

\section{PROPOSITION 4 ( Correctness I)}

Let $\tau_{1}$ be a computation tree of $H \vdash_{\Delta} s \in U$ and $H B$ its hypothesis tree.

The hypothesis tree $H B$ is evaluated with the hypothesis set $H$ to true, iff $H \vdash_{\Delta} s \in U$ has a successful subiableau.

The hypothesis tree $H B$ is evaluated with the hypothesis set $H$ to false, iff $H \vdash_{\Delta} s \in U$ has no successful subtableau.

Proof: omitted in this version

\section{PROPOSITION 5 ( Correctness II)}

Let $H B$ be the hypothesis tree of a sequent $H_{1} \vdash_{\Delta} s \in U$, and $H_{2}$ a hypothesis set.

If $\mathrm{HB}$ together with $\mathrm{H}_{2}$ evaluates to true, then the sequent $\mathrm{H}_{2} \vdash_{\Delta} s \in U$ has a successful subtableau.

If $H B$ together with $H_{2}$ evaluates to false, then the sequent $H_{2} \vdash_{\Delta} s \in U$ has no successful subtableau.

Proof: omitted in this version 


\section{$7 \quad$ Benchmarks}

The presented algorithm is implemented in QUINTUS-PROLOG on a SUN/SPARC system.

In the following examples the standard tableau model checker is compared to the tableau recycling model checker. As units of measurement we took the number of nodes in the computation tree and the system time which the model checker took to solve the task.

Examples 2 to 5 from section 4, Fig.1:

\begin{tabular}{|c|c|c|c|c|}
\hline & \multicolumn{2}{|c|}{$\begin{array}{l}\text { standard tableau model checker } \\
\text { number of nodes | time }\end{array}$} & \multicolumn{2}{|c|}{$\begin{array}{l}\text { tableau recycling model checker } \\
\text { number of nodes time }\end{array}$} \\
\hline $\mathrm{n}=3, \mathrm{k}=3$ & 105 & $<1 \mathrm{~s}$ & 25 & $<1 \mathrm{~s}$ \\
\hline $\mathrm{n}=4, \mathrm{k}=3$ & 211 & $1 \mathrm{~s}$ & 31 & $<1 \mathrm{~s}$ \\
\hline$n=3, k=4$ & 321 & $1 \mathrm{~s}$ & 33 & $<1 s$ \\
\hline$n=4, k=4$ & 851 & $2 \mathrm{~s}$ & 41 & $<1 s$ \\
\hline
\end{tabular}

Example $6 \quad: \vdash 1 \in \nu Z .(a\rangle \mu X .\langle a\rangle\langle a\rangle X \wedge\langle a\rangle\langle a\rangle Z$

\begin{tabular}{|l|l|l|l|}
\hline $\begin{array}{l}\text { standard tableau model checker } \\
\text { number of nodes }\end{array}$ & \multicolumn{2}{|c|}{$\begin{array}{l}\text { tableau recycling model checker } \\
\text { number of nodes }\end{array}$} & time \\
\hline 32766 & $74 \mathrm{~s}$ & 139 & $4 \mathrm{~s}$ \\
\hline
\end{tabular}
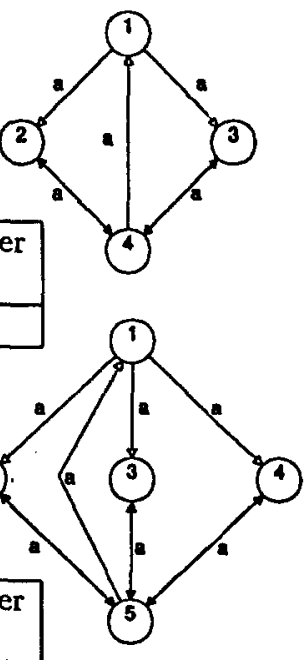

Example $\gamma \quad: \vdash 1 \in \nu Z .\langle a\rangle \mu X .\langle a\rangle\langle a\rangle X \wedge\langle a\rangle\langle a\rangle Z$

\begin{tabular}{|c|c|c|c|}
\hline \multicolumn{2}{|c|}{ standard tableau model checker } & \multicolumn{2}{|c|}{ tableau recycling model checker } \\
\hline number of nodes & time & number of nodes & time \\
\hline$>22100000$ & $>1.5 \mathrm{~h}$ & 218 & $1326 \mathrm{~s}$ \\
\hline
\end{tabular}

\section{Conclusion}

A tableau based model checker for the full modal mu-calculus was presented, which profits from the idea to recycle subtableaux which have been calculated earlier in the model checker algorithm. The information contained in a subtableau is reduced to a much smaller expression. An implementation of this algorithm showed in several examples an impressive acceleration.

Future work will include the following aspects:

We continue to get more experience with real world transition systems and relevant modal properties when verified with the tableau recycling model checker and different model checking approaches.

Secondly in this paper an idea was worked out how the maximal informatation can be 
preserved during a proof run. Heuristic methods could help to do it without maximal information in order to reduce memory expense.

Finally we will continue in investigating the complexity of the model checker algorithm.

Acknowledgement I thank Dirk Taubner for many motivating discussions. Florian Mengedoht implemented the algorithm.

\section{$9 \quad$ References}

[B] Julian Bradfield, Verifying Temporal Properties of Systems, Birkhäuser,1992.

[BC] J.R.Burch, E.M.Clarke, K.L.McMillan, D.L.Dill and L.J.Hwang, Symbolic model checking: $10^{20}$ states and beyond, in: Information and Computation, Vol 98, Num 2, June 1992, (141-170).

[BS] Julian Bradfield and Colin Stirling, Verifying Temporal Properties of Processes, CONCUR 1990, in: LNCS 458, Springer Verlag, Berlin, 1991, (115-125).

[C] Rance Cleaveland, Tableau Based Model Checking in the Propositional MuCalculus, in: Acta Informatica,1990,(725-747).

[CS1] Rance Cleaveland and Bernhard Steffen, A Linear-Time Model-Checking Algorithm for the Alternation-Free Modal Mu-Calculus, in: Proc. of the Third Workshop on Compuler Aided Verification, LNCS 575, 1992,(48-58).

[CS2] Rance Cleaveland and Bernhard Steffen, Computing Behavourial Relations, Logically, in: Proc. ICALP $91,1991$.

[EFT] Reinhard Enders, Thomas Filkorn, Dirk Taubner, Generating BDDs for Symbolic Model Checking in CCS, in: Proc. of the Third Workshop on Computer Aided Verification, LNCS 575, 1992, (203-213).

[EL] E.Allen Emerson and Ching-Luang Lei, Efficient model checking in fragments of the propositional mu-calculus, in: Proc. of Symposium on Logic in Computer Science,IEEE, 1986, (267-278).

[S] Colin Stirling, Modal and Temporal Logics, in: S.Abramsky, D.Gabbay, and T.Maibaum, editors, Handbook of Logic in Computer Science, Oxford University Press.

[SW] Colin Stirling and David Walker, Local model checking in the modal mu-calculus, in: Proc. International Conference on Theory and Practice of Software Development, LNCS 351, Springer Verlag, Berlin,1989,(369-382). 